\title{
THE TREATMENT OF RHEUMATOID ARTHRITIS DURING PRECONCEPTION AND PREGNANCY
}

\author{
Anca Boca ${ }^{1,2}$, Ioan Ancuta ${ }^{1,2}$, Florin Bobirca ${ }^{3}$, Cristina Tataru ${ }^{1}$, Cristina Comsa ${ }^{1}$, Carina Mihai ${ }^{1,2}$, \\ Mihai Bojinca $^{1,2}$, Victor Stoica ${ }^{1,2}$ \\ ${ }^{1}$ Internal Medicine and Rheumatology Department, Dr. I. Cantacuzino Clinical Hospital, Bucharest, \\ Romania \\ ${ }^{2}$ Faculty of Medicine, Carol Davila University of Medicine and Pharmacy, Bucharest, Romania \\ ${ }^{3}$ General Surgery Department, Dr. I. Cantacuzino Clinical Hospital, Bucharest, Romania
}

\begin{abstract}
Regarding the pregnancy outcome in patients with Rheumatoid Arthritis (RA), most concerns are linked to pharmacological therapies. In order to manage the disease and obtain a favourable outcome of the pregnancy, there are several remissive or biological therapies that may be used, with minimal precautions.

The anti-rheumatic drugs that may be used in RA are classified into 4 pregnancy risk categories according to the risk of inducing fetal injury: minimal risks, medium risks, high risks, and unknown risks.

For many biological therapies, the lack of extensive studies is limiting the therapeutic recommendations in this sensitive area; recommendations are coming from experts in this field.

However, if the preconceptual period and all the pregnancy conducts comply with the general recommendations, we may conclude that medication and pregnancy in rheumatoid arthritis may be combined with minimal risks.
\end{abstract}

Keywords: pregnancy, rheumatoid arthritis, anti-rheumatic drugs

Rheumatoid arthritis (RA) is an autoimmune rheumatic disease that does not have absolute contraindications to pregnancy.

Most concerns are linked to medication and teratogenicity, most probably due to the lack of studies on an extended population, which is impossible to achieve under ethical medical conditions. Nevertheless, based on clinical cases and multinational observational studies, there is enough data that can orient the therapeutic conduct in the preconception phase, as well as during pregnancy and breastfeeding in patients with RA.

The success of a pregnancy is primarily due to good therapeutic management in the preconception phase, to adequate planning, which must include an efficient control of the disease (low disease activity or remission according to ACR/EULAR criteria of remission, at least 6 months prior to conception) and discontinuation of foetal risk medication on a timely and effective manner as recommended by international experts.

\section{Anti-rheumatic therapy}

Depending on the potential adverse effects and toxicity of the anti-rheumatic drugs administered during pregnancy and breastfeeding, these have been classified into 4 categories (1):

1. Minimal risks: Hydroxychloroquine, Sulfasalazine, Intravenous Immunoglobulins, Heparin and Low Molecular Weight Heparin

2. Medium risks: Aspirin and Nonsteroidal Anti-Inflammatory Drugs (NSAIDs), Glucocorticoids, Azathioprine, Cyclosporine, Colchicine, Anti TNF Alpha Therapy

3. High risks: Methotrexate, Leflunomide, Mycophenolate mofetil, Cyclophosphamide

4. Unknown risks: Rituximab, Abatacept, Anakinra, Tocilizumab, Belimumab

Given the classification shown above, rheumatologists may use the table below in order to apply good management of medication in RA, as well as in other autoimmune diseases sharing the same thera- 
py, in the preconceptual period and during the pregnancy.

TABLE 1. Use of anti-rheumatic drugs during pregnancy, according to the pregnancy risk (2)

\begin{tabular}{|c|c|}
\hline $\begin{array}{l}\text { Drugs to be } \\
\text { discontinued before a } \\
\text { planned pregnancy }\end{array}$ & $\begin{array}{l}\text { Methotrexate, Leflunomide, } \\
\text { Rituximab, Tocilizumab, Abatacept, } \\
\text { Cyclophosphamide, Mycophenolate } \\
\text { mofetil }\end{array}$ \\
\hline $\begin{array}{l}\text { Drugs to be } \\
\text { discontinued during } \\
\text { the first trimester }\end{array}$ & $\begin{array}{l}\text { Golimumab, Ustekinumab, } \\
\text { Belimumab }\end{array}$ \\
\hline $\begin{array}{l}\text { Drugs to be } \\
\text { discontinued at the } \\
\text { end of the second } \\
\text { trimester }\end{array}$ & $\begin{array}{l}\text { Non-Selective Anti-Inflammatory } \\
\text { Drugs, Infliximab, Adalimumab, } \\
\text { Etanercept }\end{array}$ \\
\hline $\begin{array}{l}\text { Drugs that can be used } \\
\text { throughout pregnancy }\end{array}$ & $\begin{array}{l}\text { Sulfasalazine, Hydroxychloroquine, } \\
\text { Low Dose Aspirin, Paracetamol, } \\
\text { Certolizumab, Prednisone, } \\
\text { Prednisolone, Cyclosporine, } \\
\text { Azathioprine, Intravenous } \\
\text { Immunoglobulin, Chloroquine, } \\
\text { Tacrolimus }\end{array}$ \\
\hline
\end{tabular}

According to the EULAR 2015 recommendations (3), anti-rheumatic therapy in a planned pregnancy in patients with RA must be discontinued by a various number of weeks, as follows:

- Methotrexate: discontinuation 12 weeks before conception

- Leflunomide: discontinuation and effective washout (with cholestyramine)

- Mycophenolate mofetil: discontinuation 6 weeks before conception

- Abatacept: discontinuation 12 weeks before conception

- Tocilizumab: discontinuation 12 weeks before conception

- Anti TNF Alpha - discontinuation at the time of the diagnosis of pregnancy, when the pregnancy test becomes positive. In case of an active and insufficiently controlled disease by synthetic medication, except for Certolizumab pegol, that may be used over the entire period of pregnancy, the rest of the TNF Alpha inhibitors must be discontinued between gestational weeks 20 to 30 .

- Bisphosphonates: discontinuation upon conception

In order to gain a better understanding of all the recommendations listed above, we shall singularize, for each medication, their direct involvement in the foetal or gestational pathology.

\section{NSAIDs}

According to their classification, they are average risk drugs, therefore can be used during pregnancy, and the experience is sufficient enough, the limitations pertaining more to preconception and the final trimester of pregnancy.

It is recommended to interrupt NSAIDs in the preconception phase, at least one ovulating cycle before conception, because, as recent studies have shown - one of which has been carried out in Romania (4) - it has been demonstrated that anti-inflammatory medication inhibits the ovary follicle rupture, resulting in anovulatory cycles leading to decrease in fertility.

Moreover, it has been proven that selective Cox 2 medication does not only inhibit ovulation, but also limits the implantation of the fertilized egg, therefore having a double implication in subfertility or infertility. $(5,6)$

The use of NSAIDs during the first trimester of pregnancy did not increase the risk of foetus malformation, however it has led to an increase in spontaneous abortions. As far as using anti-inflammatory medication during trimesters 2 and 3 is concerned, warnings appear in relation to the foetal renal suffering and during trimester 3 , and after week 32 NSAIDs are strictly contraindicated due to the risk of premature closure of the arterial duct, which may lead to pulmonary arterial hypertension in the foetus. (7)

The only molecule of the NSAIDs class allowed over the entire duration of pregnancy remains Aspirin, which in low, anti-platelet dose, has been proven to prevent preeclampsia and eclampsia, if administered starting from the first trimester of pregnancy. (8).

\section{Glucocorticoids}

Glucocorticoids (GC) are allowed during pregnancy and in the preconception phase under any form and in various dosages, ideally less than the equivalent of $15 \mathrm{mg}$ /day of oral Prednisone.

The administration of GC during the first trimester of pregnancy, more precisely no later than the weeks 12-14, has been associated in older studies with an increased risk of palatoschisis (relative risk 3.4), however, recent surveys have been unable to prove such a high rate. $(9,10)$.

Prednisone and Methylprednisolone, more frequently used in rheumatic diseases, are non-fluori- 
nated products, metabolized by the placenta and arrive in the foetal circulatory system in low concentrations, unlike dexamethasone and betamethasone, which cross the placenta in high concentrations and are effective for enhancing the pulmonary maturation of foetuses in pregnancies with a very high risk of prematurity.

Therefore, in order to control the mother's disease we shall choose hydrocortisone or prednisone, whereas, when indicated to use GC with a foetal effect, dexamethasone or betamethasone should be chosen.

While the myth of palatoschisis begins to decrease also in terms of impact, an increased attention must go towards the increased prematurity rate and intrauterine growth deficit for foetuses which were exposed in utero to cortisone, as well as the maternal risks: high blood pressure, diabetes mellitus, osteopenia. (7)

\section{Hydroxychloroquine}

Antimalarial drugs do not pose special risks to pregnancy. Although initially red flags were raised in relation to their foetal ocular and auditive toxicity, they are drugs allowed for the entire duration of the pregnancy. The experience of administration comes from pregnant patients with systemic lupus erythematosus, where hydroxychloroquine (HCQ) prevents the intrapartum flare risk and decreases by as much as 3 times the risk of high degree atrioventricular block in the foetus, in the context of presence of anti-Ro and anti-La antibodies (11). Moreover, the dose of HCQ allowed during pregnancy does not show any restrictions, and it is accepted between 200 and $400 \mathrm{mg} /$ day.

\section{Sulfasalazine}

Sulfasalazine (SSZ) is a potent non-competitive inhibitor of the reduced folate carrier, the dominant cell membrane transporter for natural folates and also for methotrexate. It has been suspected that SSZ may increase by 2 to 3 times the risk of palatoschisis and of cardiovascular anomalies in exposed foetuses, therefore it requires precaution and at the same time supplementation of Folic Acid before conception and during pregnancy. $(7,12)$. Impairment on haematopoiesis as part of a prenatal exposure to SSZ has been reported in only one case (foetal haemolytic anaemia, aplastic anaemia and neutropenia). (7)

Subsequent observational surveys have not confirmed the increase of foetal risks associated with the exposure to SSZ and it is currently accepted to use SSZ on the entire duration of pregnancy if the disease requires it and up to the dosage of $2 \mathrm{~g} /$ day, at the same time being mindful of the prophylactic dosage of folic acid $5 \mathrm{mg}$ daily.

\section{Azathioprine}

Experience with AZA during pregnancy is more extensive in gastroenterology, in patients suffering from inflammatory bowel disease. Although it was recently suggested that there was a slightly increased risk of atrial or ventricular septal defects with AZA exposure, this should be weighed against possible consequences of discontinuing AZA, such as disease activation, during pregnancy. (13) The risk of foetal malformation after exposure to AZA has been found to be similar to the risk in the general population, and the recommended dosage for AZA in pregnancy is below $2 \mathrm{mg} / \mathrm{kg} /$ day. Of course, AZA is not a firstline and not even a $2^{\text {nd }}$ line therapy for RA, but it could be an option during pregnancy.

\section{Methotrexate}

A folate cycle inhibitor, methotrexate (MTX) is the largest recognized teratogen of all the anti-rheumatic medications, especially if used during the first trimester of pregnancy and in dosages of more than $10 \mathrm{mg} /$ week. Aside from the teratogenic risk, MTX is also recognized to produce a high abortive effect during the first trimester of pregnancy, the rate of spontaneous abortion having values of up to $46 \%$. (14)

The critical period of exposure of the foetus to MTX is considered to be until the weeks 6-8 of the pregnancy, respectively the period of organogenesis, in which case multiple malformations may occur, such as: pulmonary atresia, anomalies of the central nervous system - anencephaly, hydrocephaly, meningo-myelopathy, craniosynosthosis and skeletal anomalies (of the limbs). (14)

It is recommended to discontinue MTX at least 12 weeks prior to conception and to supplement folic acid.

\section{Leflunomide}

This is a drug recognized as teratogenic according to studies performed on animals; its teratogenicity has not been proven on human beings. There are 2 studies published by the OTIS (Organization of Teratology Information Specialists), performed on pa- 
tients with exposure to Leflunomide periconceptually and in pregnancy, which have shown that the malformation rate has been three times higher than in the general population. (15) However, the malformations identified have been minor, for the most part, compatible with survival.

It is generally recommended to discontinue Leflunomide (LEF) 2 years before a planned pregnancy, or to perform a wash-out by cholestyramine ( 8 grams 3 times a day, over 11 days). Regardless of the method chosen, ideally, there must be a check of the LEF serum concentration - compatible with pregnancy is a concentration lower than $0,02 \mathrm{mg} / \mathrm{ml}$. (16)

\section{Cyclophosphamide}

It is recommended to discontinue cyclophosphamide (CYC) at least 12 weeks prior to a planned pregnancy, and the risk of foetal malformation occurrence is highest if the foetus is exposed during the first trimester of pregnancy. In cases when it was necessary to resume the therapy with CYC in the $2^{\text {nd }}$ and $3^{\text {rd }}$ trimester of pregnancy, foetal cytopenia was noticed. (17)

CYC is also known to decrease fertility; young female patients undergoing treatment with $\mathrm{CYC}$ are sometimes given hormone therapy in order to preserve ovary function. The use of CYC for treatment of RA is restricted to cases refractory or intolerant to all first-line and second line disease-modifying agents, so the exposure to CYC periconceptually and during the pregnancy has been quite limited.

\section{BIOLOGICAL THERAPY IN RHEUMATOID ARTHRITIS AND PREGNANCY}

\section{Anti TNF-alpha therapy}

The exposure to anti TNF alpha therapy in pregnancy has not been proven to increase the risk of congenital malformations. The general recommendation when using TNF inhibitors (TNFi) is to discontinue treatment at the beginning of pregnancy (when the pregnancy test confirms the pregnancy).

All types of TNFi cross the placenta in variable concentrations, varying according to the stage of pregnancy, most of them during the $2^{\text {nd }}$ and $3^{\text {rd }}$ trimester, respectively, and once arrived in the foetal blood they can predispose the newly born to infections, due to the immunosuppression caused by TNFi. This is why even in very active RA, pregnant patients should discontinue TNFi in the $3^{\text {rd }}$ trimester of pregnancy.
Infliximab, Adalimumab and Golimumab, all IgG1 molecules, are crossing the placenta - as in pregnancy the only immunoglobulins that cross the placenta are of the IgG type and bind selectively through the Fc domain to the receptor on the surface of the syncytiotrophoblast. Etanercept is a fusion protein combining the TNF receptor region to the constant (Fc) end of the IgG1 antibody, so it crosses the placenta by the same mechanism as the abovementioned TNFi (Table 2). Similarly, Tocilizumab, Rituximab and Abatacept, having an Fc domain, cross the placental barrier like the TNFi.

Studies of the TNFi could not prove an increase of spontaneous abortions or malformations, the only warning concerning the in utero exposure during the $2^{\text {nd }}$ and $3^{\text {rd }}$ trimester of pregnancy comes from vaccination of the new-born with live vaccines or attenuated live vaccines. The literature is quoting a case of disseminated TB with fatal outcome in a new-born after having a BCG vaccination, therefore it is recommended to delay the vaccination by at least 6 months in a baby that has been exposed in utero during trimesters 2 and/or 3, or until the serum titre of the TNFi becomes undetectable. (18)

Evaluation over a 2-years duration of children who had been exposed intrapartum to TNFi has shown a good prognosis, with normal growth and development, and a favourable response to vaccination, without any significant differences when compared to foetuses of mothers suffering from inflammatory rheumatic diseases but who were not exposed in utero to TNFi. The only exception were infections, which have been more frequent in these children during their first year of life, but with a favourable outcome. (19)

TABLE 2. Transplacental passage of TNF inhibitors in the $3^{\text {rd }}$ trimester of pregnancy

\begin{tabular}{|l|l|}
\hline Drug & Median \% of maternal to cord \\
\hline Infliximab & $160 \%$ \\
\hline Adalimumab & $179 \%$ \\
\hline Certolizumab & $3.9 \%$ \\
\hline Etanercept & $7-30 \%$ \\
\hline
\end{tabular}

Mahadevan $U$ et al. Placental Transfer of Anti-Tumor Necrosis Factor Agents in Pregnant Patients with Inflammatory Bowel Disease (20)

\section{Infliximab}

Data regarding this drug are coming from studies performed for inflammatory bowel diseases as well as rheumatic diseases. As for all TNFi, medication should be stopped at the time of diagnosing the pregnancy. However, if the disease requires continuing 
the therapy with TNFi, Infliximab is not to be elected, because it crosses the placenta in a significant concentration.

\section{Adalimumab}

The OTIS (non-for-profit Organization of Teratology Information Specialists) Research Group has prospectively examined between 2004 and 2013 a number of 74 pregnant patients with RA who have been exposed to Adalimumab during pregnancy; all the mothers have received the medication during the first trimester and approximately half of them (43\%) have received it during all 3 trimesters. The malformations, anomalies, premature births, intrauterine growth deficits have been superimposable to the data obtained in other 2 groups which have been analysed, one of patients with RA without exposure to TNFi and the other of healthy women. $(19,21)$

\section{Golimumab}

There are no extensive surveys for Golimumab, so information is available only from experts' opinion and published clinical cases. As in every other TNFi, the rule of discontinuation at the time of having a positive pregnancy test should be observed, and use during pregnancy should be avoided.

\section{Certolizumab}

Certolizumab pegol (CZP) is a molecule PEGylated at the level of the Fab segment; it is missing the Fc portion, therefore it cannot be actively transported through the placenta. CZP passes through to the foetus only by means of a passive mechanism and in an extremely low percentage, therefore it is eligible for the treatment of RA over the entire duration of pregnancy, if the disease is not sufficiently controlled by means of synthetic DMARD medication. In these cases, CZP remains the only anti TNF alpha allowed over the entire duration of the pregnancy, as all other TNFi must be discontinued around weeks 20-30 because of the increased risk of infection in the new-born.

\section{Etanercept}

Etanercept is the second choice of medication during pregnancy among all the TNFi, because of its low rate of placental passage. There are extensive prospective surveys that have not been able to demonstrate any increase in teratogenicity, nor in premature birth risk or intrauterine growth deficit. How- ever, with regard to exposure in utero to Etanercept, a first case of VACTERL (Vertebral anomalies, Anal atresia, Cardiac defects, Tracheo-esophageal fistula and/or Esophageal atresia, Renal and Radial anomalies and Limb defects) syndrome in the foetus was published, and otherwise more elements belonging to the VACTERL syndrome have been seen in foetuses exposed in utero to TNFi.

Etanercept and, to the same degree, the other TNFi, may increase the risk of occurrence of malformations described under the acronym VACTERL or VATER, but one has to take into account the possibility of a confounding effect, as in most cases the patients had also been taking other medications during pregnancy. (22)

In Romania, none of the biological therapies have indication during pregnancy, in accordance with the national protocol.

\section{Rituximab}

Rituximab is recommended to be discontinued one year before any planned pregnancy, however recently acquired data limit this period to approximately 6 months. Although exposure to Rituximab before conception and during the first trimester of pregnancy has not proved an increase in the teratogen risk, nor in the infections risk, it is still recommended to discontinue it before conception, as a general rule. In case of exposure during the $2^{\text {nd }}$ or $3^{\text {rd }}$ trimester of pregnancy, there have been cases of foetal transitory lymphopenia. $(23,24)$

\section{Tocilizumab}

Tocilizumab is recommended to be discontinued at least 3 months before any planned pregnancy in all patients with RA. It is to be noticed that Tocilizumab reduces the efficacy of oral contraceptives. (25)

\section{Abatacept}

For both Rituximab and Abatacept, the experience is based on published clinical cases. Used during the first trimester of pregnancy, none of them could be proven to cause direct toxic effects, as the placental transfer was low during the first weeks. Only in case of a significant maternal benefit it is allowed to use these medications during pregnancy, nevertheless by assuming potential foetal risks, small or maybe still unidentified. (26) 


\section{CONCEPTION AND PREGNANCY PLANNING IN RHEUMATOID ARTHRITIS}

In accordance with the latest evaluation by experts in the EULAR Task "Points to Consider for use of Antirheumatic Drugs Before and During Pregnancy and Lactation", in RA the best moment to modulate medication is still before conception or at least at the time of conception. However, we must keep in mind that at any time during pregnancy there are short- or long-term solutions to overcome a reactivation of the disease, allowing at the same time a favourable outcome of the pregnancy and of the foetus.(27)

When the disease is early and/or active, the recommendation is to postpone pregnancy and to treat the disease (e.g. with methotrexate, and maybe biological agents, until substantial improvement is obtained). At that moment (low disease activity or remission of the disease) the strategy is to switch on medication compatible with pregnancy and to continue this medication several months before pregnancy and even during pregnancy.

In severe cases in treatment with TNFi, if the pregnancy occurs when the disease is still uncontrolled, it is not mandatory to discontinue at the time of confirmation by the pregnancy test. Moreover, adding synthetic drugs compatible with pregnancy can help to diminish the activity of the disease. It is important to remind that TNFi must be discontinued at least during weeks 20-30 of pregnancy, except for Certolizumab, which is allowed over the entire duration of gestation.
A new flare during pregnancy, as synthetic conventional DMARDs have a long time to come to action (2 to 3 months), only glucocorticoids, orally or intra-articularly, and/or biological therapy may rapidly control the activity of the disease. For this reason we must observe the recommendation to maintain a synthetic DMARD before conception and over the entire duration of pregnancy and breastfeeding, especially in cases that show the presence of autoimmunity (positive Auto Antibodies), cases with a negative prognosis - erosive disease, or RA that is active before conception.

Finally, every rheumatologist should modulate the medication of their patients by taking into account the national recommendations, their own choice and their patient's choice, and maybe even the patient's family choice in extenso.

\section{CONCLUSIONS}

RA is a disease permissive to pregnancy. Among anti-rheumatic medication, more or less specific to RA, there are sufficient therapeutic alternatives that do not pose additional risks to mothers and foetuses.

The key to a positive outcome of pregnancy and of RA during pregnancy is first and foremost to have a good therapeutic planning before conception and at any time during the pregnancy, to have a good interdisciplinary cooperation (general practitioner, rheumatologist, obstetrician, neonatologist, anaesthesiologist) and a high degree of adherence by the pregnant patient to all the therapeutic recommendations.

\section{REFERENCES}

1. Sammaritano L.R., Bermas B.L. Contraception and Pregnancy in Patients with Rheumatic Disease, Springer 2014: 273-284.

2. Williams M., Chakravarty E.F. Rheumatoid arthritis and pregnancy: impediment to optimal management of both biologic use before, during and after pregnancy Curr Opin Rheumatol 2014, 26:341-346

3. Guettrot-Imbert G., Costedoat-Chalumeau N., Tincani A. Pregnancy in Rheumatic Diseases and the Antiphospholipid Syndrome. In EULAR Textbook on Rheumatic Diseases (2nd edition, 2015), 577-605.

4. Micu M.C., Micu R., Ostensen M. Luteinized unruptured follicle syndrome increased by inactive disease and selective cyclooxygenase 2 inhibitors in women with inflammatory arthropathies. Arthritis Care Res. 2011, 63(9):1334-38.

5. Mendonca L.L., Khamashta M.A., Nelson-Piercy C., Hunt B.J., Hughes G.R. Non-steroidal antiinflammatory drugs as a possible cause for reversible infertility. Rheumatology (Oxford).2000; 39(8):880-2.
6. Uhler M.L., Hsu J.W., Fisher S.G., Zinaman M.J. The effect of nonsteroidal anti-inflammatory drugs on ovulation: a prospective, randomized clinical trial. Fertil Steril. 2001;76(5):957-61.

7. Ostensen M. et al. Anti-inflammatory and immunosuppressive drugs and reproduction Arthritis Res Ther. 2006; 8:209

8. Trivedi N.A. A meta-analysis of low-dose aspirin for prevention of preeclampsia. J Postgrad Med. 2011 Apr-Jun; 57(2):91-5.

9. Park-Wyllie L., Mazzotta P., Pastuszak A., Moretti M.E., Beique L., Hunnisett L., et al. Birth defects after maternal exposure to corticosteroids: prospective cohort study and meta-analysis of epidemiological studies. Teratology. 2000; 62(6):385-92.

10. Bay Bjorn A.M., Ehrenstein V., Holmager Hundborg H., Aagaard Nohr E., Toft Sorensen H., Norgaard M. Use of corticosteroids in early pregnancy is not associated with risk of oral clefts and other congenital malformations in offspring. Am J Ther. 2012; 21(2):73-80.

11. Brucato A., Previtali E., Ramoni V., Ghidoni S. Arrhythmias Presenting in Neonatal Lupus, Scand J Immunol. 2000; 72 (9):198-204. 
12. Hernandez-Diaz S., Werler M.M., Walker A.M.\& Mitchell A.A. Folic acid antagonists during pregnancy and the risk of birth defects. N Engl J Med. 2000; 343: 1608-14.

13. Norgard B., Pedersen L., Fonager K., Rasmussen S.N., Sorensen H.T. Azathioprine, mercaptopurine and birth outcome: a population-based cohort study. Aliment Pharmacol Ther 2003; 17: 827-34.

14. Hyoun S.C., Obican S.G., Scialli A.R. Teratogen update: methotrexate. Birth Defects Research Part A. Clin Mol Teratol. 2012; 94: 187-207

15. Cassina M., Johnson D.L., Robinson L.K., et al. Pregnancy outcome in women exposed to leflunomide before or during pregnancy. Arthritis Rheum 2012; 64:2085-2094.

16. Bonnie L. Bermas, Non-steroidal anti-inflammatory drugs, glucocorticoids and disease modifying anti-rheumatic drugs for the management of rheumatoid arthritis before and during pregnancy. Curr Op Rheumatol. 2014; 26 (3): 334-40.

17. Soh M.C.; Nelson-Piercy C. High-risk Pregnancy and the Rheumatologist. Rheumatology. 2015; 54(4):572-87.

18. Cheent K, Nolan J., Shariq S., Kiho L., Pal A., Arnold J. Case Report: Fatal case of disseminated BCG infection in an infant born to a mother taking infliximab for Crohn's disease. J Crohns Colitis. 2010 Nov; 4(5):603-5.

19. Ostensen M., Andreoli L., Brucato A., Cetin I., Chambers C., Clowse M.E. at al. State of art: Reproduction and pregnancy in rheumatic diseases. Autoimmun Rev. 2015 May; 14(5):376-86.

20. Mahadevan U., Wolf D.C., Dubinsky M., Cortot A., Lee S.D., et al. Placental transfer of anti-tumor necrosis factor agents in pregnant patients with inflammatory bowel disease. Clin Gastroenterol Hepatol. 2013 Mar; 11(3):286-92.

21. Chambers C., Johnson D.L., Luo Y. et al. Pregnancy Outcome in Women Treated with Adalimumab for the Treatment of Rheumatoid ArtTreatment of Rheumatoid Arthritis. Arthritis Rheumatol 2014; 66:10 (Supplement), S 361 (abstract)

22. Diav-Citrin O., Otcheretianski Volodarsky A., Shechtman S., Ornoy A. Pregnancy outcome following gestational exposure to TNF-alpha-inhibitors: a prospective, comparative, observational study. Reprod Toxicol. 2014 Jan; 43:78-84

23. Ostensen M. and Forger F. How safe are anti-rheumatic drugs during pregnancy? Current Opinion in Pharmacology 2013, 13:470-475

24. Klink D.T., van Elburg R.M., Schreurs M.W., van Well G.T. Rituximab administration in third trimester of pregnancy suppresses neonatal B-cell development. Clin Dev Immunol. 2008; 2008:271-363.

25. Ostensen M. Antirheumatic drugs in pregnancy (Oral presentation) International Conference on Pregnancy, Trondheim 2014.

26. Ojeda-Uribe M., Afif N., Dahan E., Sparsa L., Haby C., Sibilia J., Ternant D., Ardizzone M. Exposure to abatacept or rituximab in the first trimester of pregnancy in three women with autoimmune diseases. Clin Rheumatol. 2013 May; 32(5):695-700

27. Skorpen A.C., Ostensen M. for the EULAR Task Force "Points to Consider for use of Antirheumatic Drugs Before and During Pregnancy and Lactation": Progress of a Eular Task Force. Ann Rheum Dis 2015; 74: 242 